\title{
Incidencia de macrocefalia neonatal en el Hospital Cayetano Heredia, 2016 - 2017
}

Incidence of neonatal macrocephaly at the Hospital Cayetano Heredia, 2016 - 2017

\author{
Carol Munayco Cortez ${ }^{1,2, \text { a }}$, Daniel Guillén Pinto ${ }^{1,2, \mathrm{~b}}$
}

\section{RESUMEN}

Objetivo: Determinar la incidencia de macrocefalia neonatal en un hospital de tercer nivel en Lima, Perú. Material y Métodos: Se definió macrocefalia en función al perímetro cefálico mayor que el percentil 97 para la edad y sexo. La información fue obtenida de los registros del Sistema Informático Perinatal del Hospital Cayetano Heredia durante el periodo $1^{\circ}$ de enero, 2016 a 31 de diciembre, 2017. Se usó la Tabla Fenton para determinar los límites de normalidad. Resultados: Se registraron 210 casos de macrocefalia neonatal y una tasa general de 26 por mil nacidos vivos, inversamente proporcional a la edad gestacional. Conclusiones: la macrocefalia es más frecuente en prematuros, con un factor de riesgo cinco veces mayor en este grupo poblacional.

PALABRAS CLAVE: Macrocefalia, neonato, incidencia, pretérmino.

\section{SUMMARY}

Objective: To determine the incidence of neonatal macrocephaly in a third level hospital from Lima, Perú. Methods: Macrocephaly was defined on the basis of a cephalic perimeter higher than the 97th percentile for age and sex. The report records of the Perinatal Computer System of Cayetano Heredia Hospital between January $1^{\text {st }}$., 2016 and December $31^{\text {st }}$, 2017 were used, as well as the Fenton growth chart to determine the limits of normality. Results: A total of 210 cases of neonatal macrocephaly was registered, a general rate of 26 per 1,000 live births. Neonatal macrocephaly incidence were inversely proportional to gestational age. Conclusions: Macrocephaly was higher in preterm neonates, with a risk factor five times higher in this population group.

KEY WORDS: macrocephaly, neonate, incidence, preterm.

\section{INTRODUCCIÓN}

El termino macrocefalia se refiere al tamaño de la cabeza significativamente más grande de lo normal. Clínicamente, se identifica cuando el perímetro cefálico es mayor de 2 desviaciones estándar o mayor del 97 percentil para la edad y el sexo (1).
En el Perú, Cabieses describió este término en las culturas preincas con frases como "el cabezón de la comunidad", persona que era apodada umasapa; aunque cuando la desproporción con el cuerpo era muy marcada, se usaba el vocablo riwihuma (2), de esta forma destaca la popularidad del conocimiento.

\footnotetext{
Universidad Peruana Cayetano Heredia. Lima, Perú.

Hospital Cayetano Heredia. Lima, Perú.

Médico Residente;

b Neurólogo Pediatra
} 
Desde el punto de vista poblacional, se espera que la macrocefalia se encuentre en el $5 \%$ de la población pediátrica (3). Sin embargo, hay escasa información sobre su prevalencia e incidencia, tanto a nivel mundial como en Latinoamérica. En el Perú, no se encuentran registros sobre macrocefalia.

La importancia de la detección neonatal destaca por la relación con la etiología subyacente, entre las que se describe una serie de causas, como macrocefalia familiar, síndromes neuroectodérmicos, cromosomopatías, malformaciones cerebrales (hidrocefalia congénita), infecciones intrauterinas (toxoplasmosis, citomegalovirus, coriomeningitis linfocitaria) e incluso raros desórdenes como las enfermedades peroxisomales (Zelweger, Canavan).

El propósito de este estudio fue determinar la incidencia de la macrocefalia neonatal en el Hospital Cayetano Heredia entre los años 2016 y 2017.

\section{MATERIAL Y MÉTODOS}

Se realizó un estudio observacional descriptivo exploratorio, utilizando el Reporte del Hospital Cayetano Heredia de Lima $(\mathrm{HCH})$ al Sistema Informático Perinatal (SIP), del 1 de enero del 2016 hasta el 31 de diciembre del 2017.

Fueron incluidos los datos de todos los recién nacidos vivos que tuvieran registro del perímetro cefálico, de 24 a 40 semanas de edad gestacional. Se seleccionaron los neonatos que tuvieron perímetro cefálico mayor del límite superior para la edad gestacional y sexo.

Para determinar los límites superiores del perímetro cefálico para neonatos prematuros y a término de 37 a 40 semanas inclusive, se usó la Curva de Crecimiento de Fenton 2013. Se registró el número de casos por sexo y edad gestacional.

El estudio contó con la aprobación del Comité de Ética del $\mathrm{HCH}$. Los datos fueron almacenados y depurados en una hoja de cálculo del programa Excel MicrosoftC, luego fueron procesados con el programa STATA $(C)$ versión 13. Se aplicaron tablas de frecuencias absolutas y porcentuales, y se determinaron las tasas de incidencia. Se aplicó el riesgo relativo (RR) para confirmar si las variables de exposición conducían a un mayor riesgo de macrocefalia. En las pruebas de inferencia se utilizó un intervalo de confianza al 95\%.

\section{RESULTADOS}

Durante el periodo de estudio, se registraron 8160 recién nacidos vivos, habiendo descontado 80 omisiones de perímetro cefálico y 101 fallecidos. Entre ellos, 210 casos de macrocefalia fueron registrados, 90 en el año 2016 y 120 en el 2017, determinando una incidencia de 26 casos por cada mil nacidos vivos en el periodo 2016-2017.

La tabla 1 muestra que la incidencia de macrocefalia en el 2017 fue de 2,9\% aumentando un $0.6 \%$ con respecto al 2016. Estas tasas de incidencia, se encontraron en relación inversa a la edad gestacional.

En el gráfico 1 se muestra la incidencia de macrocefalia según sexo y edad gestacional, se observa que la macrocefalia sólo en el intervalo de 28-32 semanas de edad gestacional fue mayor en hombres que en mujeres; pero en general, las mujeres presentaron mayor incidencia de macrocefalia.

La edad gestacional analizada desde las 24 semanas hasta las 40 semanas. La incidencia de macrocefalia en los niños a término fue de $1.8 \%$, y en los prematuros

Tabla 1. Incidencia de Macrocefalia de acuerdo a la edad gestacional. HCH, 2016-2017

\begin{tabular}{cccccccccc}
\hline & \multicolumn{3}{c}{$2016-2017$} & \multicolumn{2}{c}{2016} & \multicolumn{3}{c}{2017} \\
\hline EG (sem) & $\mathbf{n}^{*}$ & Casos & Incidencia & $\mathbf{n}$ & Casos & Incidencia & $\mathbf{n}$ & Casos & Incidencia \\
$24-27$ & 41 & 6 & $14,6 \%$ & 21 & 2 & $9,5 \%$ & 20 & 4 & $20,0 \%$ \\
$28-29$ & 25 & 9 & $36,0 \%$ & 12 & 3 & $25,0 \%$ & 13 & 6 & $46,2 \%$ \\
$30-31$ & 57 & 5 & $8,8 \%$ & 30 & 2 & $6,7 \%$ & 27 & 3 & $11,1 \%$ \\
$32-33$ & 85 & 8 & $9,4 \%$ & 33 & 3 & $9,1 \%$ & 52 & 5 & $9,6 \%$ \\
$34-36$ & 614 & 48 & $7,8 \%$ & 275 & 20 & $7,3 \%$ & 339 & 28 & $8,3 \%$ \\
$37-38$ & 2720 & 85 & $3,1 \%$ & 1,361 & 39 & $2,9 \%$ & 1,359 & 46 & $3,4 \%$ \\
$39-40$ & 4618 & 49 & $1,1 \%$ & 2,238 & 21 & $0,9 \%$ & 2,380 & 28 & $1,2 \%$ \\
Total & 8160 & 210 & $2,6 \%$ & 3,970 & 90 & $2,3 \%$ & 4,190 & 120 & $2,9 \%$ \\
\hline
\end{tabular}

$*_{\mathrm{n}}=$ total de nacidos vivos 


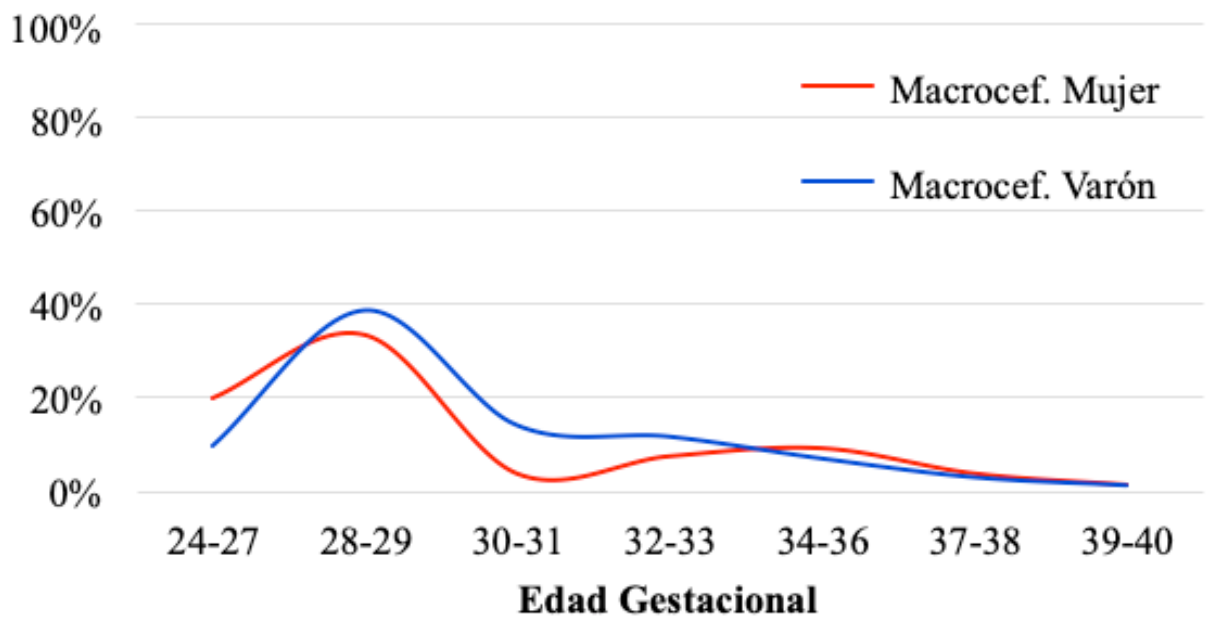

Gráfico 1. Incidencia de macrocefalia por edad gestacional y sexo. HCH, 2016-2017

Tabla 2. Macrocefalia y prematuridad según sexo. HCH, 2016-2017

\begin{tabular}{lcccccc}
\hline & \multicolumn{2}{c}{$\mathrm{PC}>\mathrm{P} 97 *$} & \multicolumn{2}{c}{$\mathrm{PC}<\mathrm{P97}$} & \\
& $\mathbf{n}$ & $\mathbf{\%}$ & $\mathbf{n}$ & $\mathbf{\%}$ & $\mathbf{R R}$ & $\mathbf{I C 9 5 \%}$ \\
\hline Mujer & & & & & & \\
Prematuras & 39 & 34,2 & 360 & 9,3 & 4,7 & $3,2-6,8$ \\
A término & 75 & 65,8 & 3,505 & 90,7 & 1 & \\
Varón & & & & & & \\
Prematuros & 37 & 38,5 & 386 & 9,4 & 5,6 & $3,7-8,3$ \\
A término & 59 & 61,5 & 3,699 & 90,6 & 1 & \\
*PC=perímetro cef́́lico, P97=percentil 97 & & & & &
\end{tabular}

Tabla 3. Causas de Macrocefalia

$\begin{array}{ll}\text { Comunicante } & \text { Acondroplasia } \\ \text { Impresión basilar } & \text { Ensanchamiento benigno del espacio subaracnoideo } \\ \text { Papiloma del plexo coroideo } & \text { Malignidad meníngea } \\ \text { Meningitis } & \text { Post hemorrágica } \\ \text { No comunicante } & \text { Abscesos } \\ \text { Estenosis del acueducto } \\ \text { Malformación Chiari } \\ \text { Malformación Dandy Walker } \\ \text { Hematoma } \\ \text { Infecciosa } \\ \text { Tumores y desórdenes neurocutáneos } \\ \text { Malformación de la vena de Galeno } \\ \text { Síndrome de Walker Warburg } \\ \text { Ligado al X } \\ \text { Hidranencefalia } \\ \text { Holoprosencefalia } \\ \text { Hidrocéfalo masivo } \\ \text { Porencefalia } \\ \end{array}$




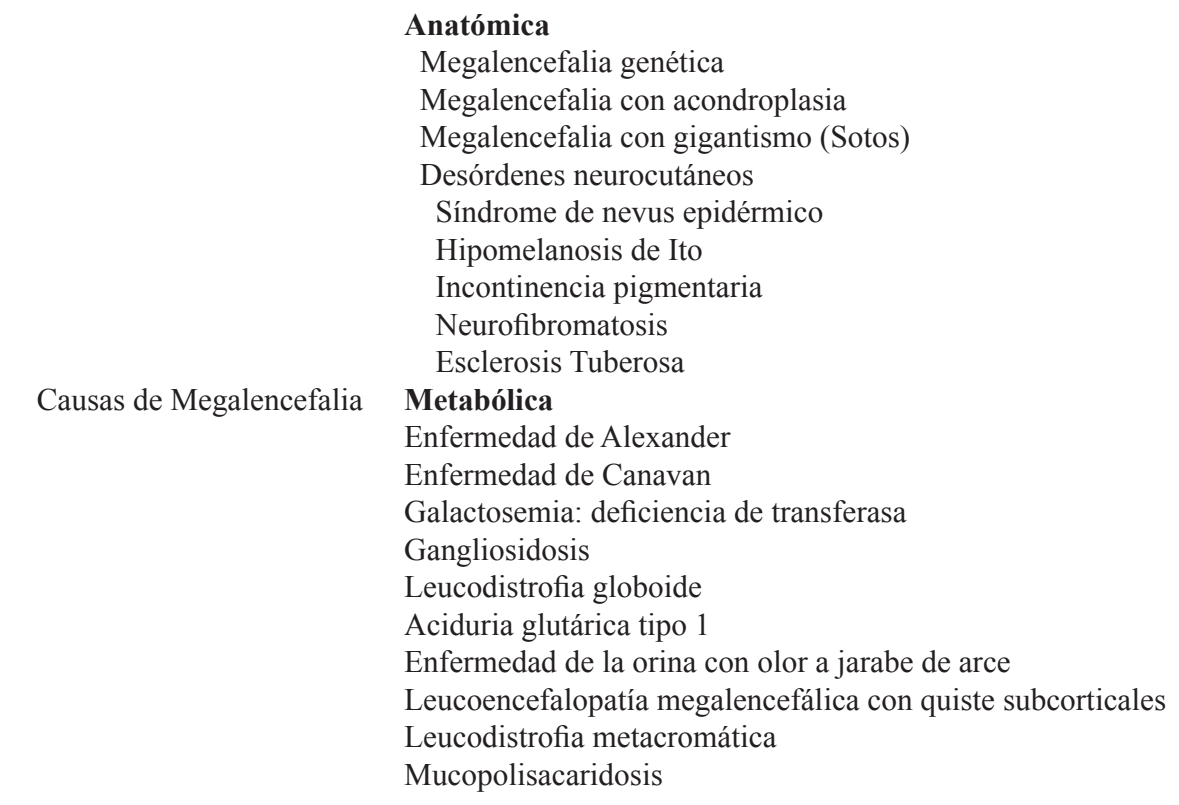

*Tomado de Clinical Pediatric Neurology: a signs and symptoms approach. Gerald M. Fenichel (4)

de $9.2 \%$. Por grupos de prematuros, se encontró $17,1 \%$ entre las 28-31 semanas y de 14,6\% entre los de 24 semanas y menores de 28 semanas.

En la tabla 2, se observa que en el grupo de mujeres con macrocefalia (PC>P97) el $34,2 \%$ son prematuras, en cambio en el grupo de mujeres sin macrocefalia son prematuras el $9,3 \%$, siendo esta diferencia porcentual significativa $(\mathrm{p}<0,001)$; un resultado similar se observó en el grupo de varones.

Las nacidas prematuras tienen 4,7 veces mayor riesgo de macrocefalia con respecto a las nacidas a término, siendo este riesgo significativo (IC95\% $=3,2$ $6,8)$ pues ambos intervalos de confianza no contienen a la unidad como muestra la tabla 3.

En el caso de los varones prematuros el riesgo relativo de macrocefalia fue 5.6, es decir, que un varón prematuro tendrá 5,6 veces más riesgo de macrocefalia con respecto a otro varón que nació a término. Y en general, los prematuros (mujeres y varones) tendrán casi 5 veces más riesgo de macrocefalia que los nacidos a término.

\section{DISCUSION}

La macrocefalia neonatal es un problema relativamente frecuente. Sin embargo, hay poca información de su incidencia y causas subyacentes. El hallazgo puede llegar a ser muy preocupante para los padres y para los médicos, dependiendo del tamaño de la cabeza.
En este estudio determinamos una incidencia que podría estar dentro del estimado estadístico. No obstante, en el trabajo de Peng Tan que toma como referencia al Centro Nacional de Percentiles Estadísticos de la Salud (NCHS) de Estados Unidos reporta un 5\% (5), estas diferencias pueden ser por la variabilidad de factores étnicos, genéticos, demográficos y socioeconómicos.

Ante un bebé con macrocefalia el primer paso es determinar si se trata de un aumento de la velocidad del crecimiento craneal o una característica persistente desde el nacimiento, situación que fácilmente se aclara siguiendo el crecimiento craneal a través de los percentiles. La causa más frecuente de macrocefalia con crecimiento craneal normal es familiar y el diagnóstico se establece si uno de los padres tiene el perímetro craneal $\geq 98 \%$; de esta definición se deduce que el $2 \%$ de la población presenta macrocefalia familiar (6).

Si ninguno de los padres tiene un perímetro craneal $\geq 98 \%$ o si existe aumento de la velocidad del crecimiento craneal es importante descartar hidrocefalia primaria o secundaria. Y cuando el infante presente retraso del desarrollo, epilepsia, rasgos dismórficos o estigmas neurocutáneos cabe sospechar que el encéfalo es grande (megalencefalia) y anormal (tabla 3). En estos casos, la hidrocefalia rápidamente se puede descartar haciendo una ecografía cerebral, que se prefiere para evitar la radiación de la tomografía, de confirmarse la hidrocefalia se podría realizar una resonancia magnética para definir el nivel 
de obstrucción y las lesiones asociadas. De tratarse de megalencefalia las causas subyacentes son muy variadas y deberá iniciarse un proceso diagnóstico al respecto $(6,7,8)$.

Particularmente, hemos analizado la frecuencia de macrocefalia de los prematuros del último trimestre de gestación. Usando las curvas de Fenton, la macrocefalia fue más frecuente en el recién nacido pretérmino, sobre todo entre las semanas 28 a 31 (17,1\%). Nuevos estudios serán necesarios para verificar si estos hallazgos observacionales son verdaderos, puesto que podrían cambiar los parámetros de normalidad.

Asimismo, el mayor número de casos de macrocefalia se encontró en las recién nacidas mujeres, con mayor diferencia en el grupo de recién nacidos extremos. Este dato, también requiere verificación en el futuro, debiendo ser tomado con cuidado puesto que el número de casos en el grupo de recién nacidos extremo fue menor y además por las condiciones de urgencia de atención podría variar el cálculo de la edad gestacional o la medición de la cabeza. En este estudio, podemos establecer que la macrocefalia neonatal fue mayor en los recién nacidos pretérmino, con un riesgo de 5 veces más en este grupo.

El presente estudio destaca por hacer énfasis en la medición del perímetro cefálico en neonatos, indicador muy eficiente del neurodesarrollo (9); y por haber establecido un sistema práctico de vigilancia de macrocefalia a través de los reportes estadísticos del SNP que todo hospital debe registrar y analizar. Asimismo, variaciones de la incidencia basal podrían advertir de nuevas enfermedades del sistema nervioso fetal. El presente estudio tiene las limitaciones de toda investigación observacional que deberá confirmarse con estudio prospectivos.

\section{CONCLUSIONES}

La incidencia de macrocefalia fue de 26 casos por cada mil nacidos vivos en el Hospital Cayetano Heredia durante los años 2016-2017. La macrocefalia estuvo en relación inversa a la edad gestacional. Las mujeres presentaron mayor incidencia de macrocefalia. La prematuridad es un factor de riesgo de macrocefalia, pues los prematuros mujeres y varones tienen 5 veces más riesgo de macrocefalia que los nacidos a término.

Fuentes de financiamiento: Autofinanciado. Conflictos de interés: Los autores no reportan ningún conflicto de interés.

\section{Correspondencia:}

Carol Giselle Munayco Cortez

Correo electrónico: carol.munayco@upch.pe

\section{REFERENCIAS BIBLIOGRÁFICAS}

1. Vertinsky AT, Barnes PD. Macrocephaly, Increased Intracranial Pressure, and Hydrocephalus in the Infant and Young Child. Top Magn Reson Imaging. 2007; 18(1):31-51.

2. Cabieses F. Apuntes de Medicina Tradicional: la racionalización de lo irracional. 2a ed. Lima: Consejo Nacional de Ciencia y Tecnología; 1993.

3. Hamill PV, Drizd TA, Johnson CL, Reed RB, Roche AF, Moore WM. Physical growth: National Center for Health Statistics percentiles. Am J Clin Nutr. 1979; 32:607-629.

4. Fenichel GM. Disorders of cranial volume and shape. En: Piña-Garza J. Clinical Pediatric Neurology. 6th ed. Philadelphia: Saunders; 2009

5. Peng AP, Mankad K, Gonçalves FG, Talenti G, Alexia E. Macrocephaly: Solving the Diagnostic Dilemma. Top Magn Reson Imaging. 2018;27(4):197-217.

6. Dunn DW, Epstein LG. Macrocefalia. En: Dunn DW, Epstein LG. Tomando decisiones en Neurología Infantil. Barcelona: Ediciones Médicas Temis SA; 1990. p. 24-25.

7. Williams CA, Dagli A, Battaglia A. Genetic disorders associated with macrocephaly. Am J Med Genet. 2008; 146A(15):2023-37.

8. Gooskens RH, Willemse J, Bijlsma JB, Hanlo PW. Megalencephaly: definition and classification. Brain Dev. 1988; 10(1):1-7

9. Medina-Alva P, Duque KR, Zea-Vera A, Bellomo S, > Cárcamo C, Guillén-Pinto $\mathrm{D}$, et al. Combined predictors of neurodevelopment in very low birth weight preterm infants. Early Human Development. 2019; 130: 109-115.

Recibido: 14/07/2019

Aceptado: 05/09/2019 